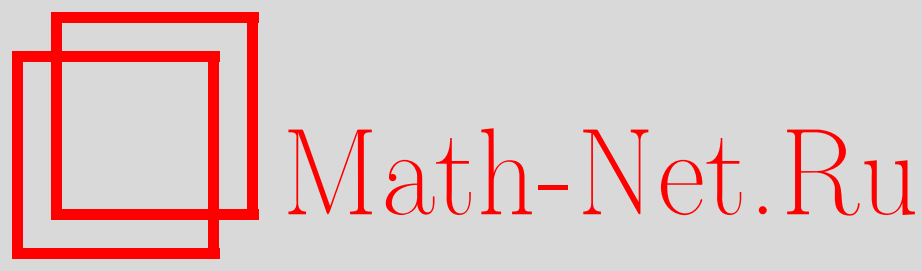

И. Т. Хабибуллин, Е. В. Гудкова, Алгебраический метод классификации $S$-интегрируемых дискретных моделей, TMФ, 2011, том 167, номер 3, 407-419

DOI: https://doi.org/10.4213/tmf6650

Использование Общероссийского математического портала Math-Net.Ru подразумевает, что вы прочитали и согласны с пользовательским соглашением http://www . mathnet.ru/rus/agreement

Параметры загрузки:

IP: 52.205 .19 .152

26 апреля 2023 г., 12:54:25

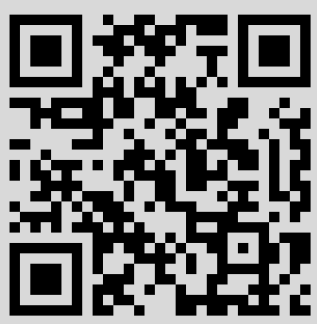




\section{АЛГЕБРАИЧЕСКИЙ МЕТОД КЛАССИФИКАЦИИ $S$-ИНТЕГРИРУЕМЫХ ДИСКРЕТНЫХ МОДЕЛЕЙ}

Обсуждается метод классификации интегрируемых уравнений на квад-графах, основанный на алгебраических идеях. Уравнению сопоставляется кольцо Ли и исследуется функция, описывающая размерность линейного пространства, линейно порожденного кратными коммутаторами генераторов кольца. В общем случае эта функция растет экспоненциально. Примеры показывают, что для интегрируемых уравнений она растет медленнее. Предложена схема классификации, основанная на этом наблюдении.

Ключевые слова: уравнения на квад-графах, классификация, характеристические векторные поля, кольцо Ли, условия интегрируемости, дискретное уравнение Кортевега-де Фриза.

\section{1. ВВЕДЕНИЕ}

В настоящее время дискретные модели (уравнения на квад-графах) вида

$$
u_{1,1}=f\left(u, u_{1}, \bar{u}_{1}\right)
$$

интенсивно изучаются в связи с их широким применением в физике, геометрии, биологии и т. Д. Поясним обозначения, использованные в (1): неизвестной является функция $u=u(m, n)$ двух независимых дискретных переменных. Нижние индексы и черта обозначают сдвиги аргументов: $u_{k}=u(m+k, n), \bar{u}_{k}=u(m, n+k), u_{1,1}=$ $u(m+1, n+1)$. Функция $f$ предполагается локально аналитической; она, вообще говоря, зависит от всех трех аргументов. Другими словами, уравнение (1) можно переписать в любом из следующих видов:

$$
u_{1,-1}=f^{1,-1}\left(u, u_{1}, \bar{u}_{-1}\right), \quad u_{-1,1}=f^{-1,1}\left(u, u_{-1}, \bar{u}_{1}\right), \quad u_{-1,-1}=f^{-1,-1}\left(u, u_{-1}, \bar{u}_{-1}\right) .
$$

Известны различные подходы к изучению интегрируемых дискретных уравнений. В работах [1]-[4] в качестве критерия интегрируемости для разностных уравнений на четырехугольных решетках было предложено свойство согласованности по

* Институт математики с вычислительным центром Уфимского научного центра РАН, Уфа, Россия. E-mail: habibullinismagil@gmail.com

†Уфимский государственный нефтяной технический университет, Уфа, Россия. E-mail: elena.gudkova79@mail.ru 
ребрам куба. Симметрийный подход к классификации интегрируемых систем был приспособлен к дискретному случаю [5]-[9]. Еще одно характеристическое свойство интегрируемого уравнения заключается в обращении в ноль его алгебраической энтропии [10]. Альтернативные методы использовались в работах [11]-[13]. В настоящей работе мы предлагаем новую схему классификации интегрируемых дискретных моделей.

Около двадцати лет назад было замечено, что характеристические алгебры Ли, введенные в работе [14], в случае интегрируемых дифференциальных уравнений в частных производных гиперболического типа, таких как уравнения синус-Гордон и Цицейки-Жибера-Шабата, обладают весьма специальным свойством. Размерность линейного пространства, натянутого на кратные коммутаторы генераторов, растет, как правило, медленнее, чем в общем случае [15]. В работе [16] обсуждалась задача строгой формализации понятия медленного роста и была высказана гипотеза, проверенная на примере классификации интегрируемых уравнений вида $u_{x, y}=f\left(u, u_{x}\right)$.

В настоящей работе мы рассматриваем другую формализацию этого свойства характеристических векторных полей и проверяем его, взяв в качестве пробного камня частный случай уравнения на квад-графах (1).

Статья построена следующим образом. В разделе 2 вводятся характеристические векторные поля и определяется пробное кольцо Ли. Выдвигается гипотеза, что имеется связь между интегрируемостью и пробным кольцом. В разделе 3 представлено описание пробного кольца для дискретного потенциированного уравнения КдФ. В разделе 4 исследована задача классификации для модели вида $u_{1,1}-u=g\left(u_{1}-\bar{u}_{1}\right)$. Результат классификации обобщен в теореме 5.

\section{2. ХАРАКТЕРИСТИЧЕСКИЕ ВЕКТОРНЫЕ ПОЛЯ И СХЕМА КЛАССИФИКАЦИИ}

Определим характеристические векторные поля для уравнения (1). Начнем с очень частного случая, когда уравнение допускает $n$-интеграл, т. е. такую функцию $I=I\left(u_{-j}, u_{-j+1}, \ldots, u_{k}\right)$, что выполнено уравнение $\bar{D} I=I$, где $\bar{D}$ - оператор сдвига: $\bar{D} h(m, n)=h(m, n+1)$. Это означает, что для любого решения $u=u(m, n)$ уравнения (1) значение функции $I$ не зависит от переменной $n$. В координатном представлении условие $\bar{D} I=I$ означает, что

$$
I\left(r_{-j+1}, r_{-j+2}, \ldots, r, \bar{u}, f, f_{1}, \ldots, f_{k-1}\right)=I\left(u_{-j}, u_{-j+1}, \ldots, u_{k}\right),
$$

где $r=f^{-1,1}\left(u, u_{-1}, \bar{u}_{1}\right)$. Очевидно, правая часть равенства (2) не зависит от $\bar{u}_{1}$, а потому выполнены условия

$$
\frac{\partial}{\partial \bar{u}_{1}} \bar{D} I=0, \quad Y I=0, \quad Y:=\bar{D}^{-1} \frac{\partial}{\partial \bar{u}_{1}} \bar{D} .
$$

С учетом формулы

$$
\frac{\partial r}{\partial \bar{u}_{1}}=\frac{1}{D^{-1}\left(\partial f / \partial \bar{u}_{1}\right)},
$$

где $D$ - сдвиг по $m, D h(m, n)=h(m+1, n)$, находим (см. также [17])

$$
Y=\frac{\partial}{\partial u}+x \frac{\partial}{\partial u_{1}}+\frac{1}{x_{-1}} \frac{\partial}{\partial u_{-1}}+x x_{1} \frac{\partial}{\partial u_{2}}+\frac{1}{x_{-1} x_{-2}} \frac{\partial}{\partial u_{-2}}+\cdots,
$$


где

$$
x=\bar{D}^{-1}\left(\frac{\partial f\left(u, u_{1}, \bar{u}_{1}\right)}{\partial \bar{u}_{1}}\right)=-\frac{\partial f^{1,-1}\left(u, u_{1}, \bar{u}_{-1}\right) / \partial u}{\partial f^{1,-1}\left(u, u_{1}, \bar{u}_{-1}\right) / \partial u_{1}} .
$$

Назовем $Y$ характеристическим векторным полем. Теперь вернемся к общему случаю и определим характеристическое векторное поле $Y$ для цепочки (1) как формальный ряд, задаваемый формулой (3).

Обозначим через $T$ множество векторных полей, полученых взятием всех возможных кратных коммутаторов и линейных комбинаций операторов $X:=\partial / \partial \bar{u}_{-1}$ и $Y$ с коэффициентами, зависящими от конечного числа динамических переменных $\bar{u}_{-1}$, $u, u_{ \pm 1}, u_{ \pm 2}, \ldots$ Ясно, что, множество $T$ имеет структуру кольца Ли. Назовем его пробным кольцом уравнения (1) в направлении $n$. Аналогично можно определить пробное кольцо $\bar{T}$ в направлении $m$.

Заметим, что для интегрируемых по Дарбу уравнений вида (17) как $T$, так и $\bar{T}$ - кольца конечной размерности. В действительности пробное кольцо является подмножеством характеристического кольца Ли [17].

Обозначим через $V_{j}$ линейное пространство над полем локально аналитических функций, натянутое на $X, Y$ и все кратные коммутаторы функций $X$ и $Y$ порядка меньше или равного $j$, так что

$$
V_{0}=\{X, Y\}, \quad V_{1}=\{X, Y,[X, Y]\}, \quad \ldots
$$

Введем функцию $\Delta(k)=\operatorname{dim} V_{k+1}-\operatorname{dim} V_{k}$. Следующая гипотеза подтверждается многочисленными примерами.

ГипотезА (алгебраический тест). Любая интегрируемая модель вида (1) удовлетворяет следующему условию: найдется последовательность натуральных чисел $\left\{t_{k}\right\}_{k=1}^{\infty}$, для которой $\Delta\left(t_{k}\right) \leqslant 1$.

Кольцо $T$ допускает автоморфизм, порожденный оператором сдвига $D$,

$$
T \ni Z \stackrel{\text { Aut }}{\rightarrow} D Z D^{-1} \in T
$$

который играет ключевую роль в наших дальнейших рассуждениях. Важно, что $X$ и $Y$, рассматриваемые как операторы на множестве функций, зависящих от переменных $\bar{u}_{-1}, u, u_{ \pm 1}, u_{ \pm 2}, \ldots$, удовлетворяют соотношениям сопряжения

$$
D X D^{-1}=p X, \quad D Y D^{-1}=\frac{1}{x} Y
$$

где

$$
p=D\left(\frac{\partial f^{-1,-1}\left(u, u_{-1}, \bar{u}_{-1}\right)}{\partial \bar{u}_{-1}}\right)=\frac{1}{\partial f^{1,-1}\left(u, u_{1}, \bar{u}_{-1}\right) / \partial \bar{u}_{-1}} .
$$

Действительно, определим коэффициенты оператора $D X D^{-1}=\sum a_{i} \partial / \partial u_{i}+p \partial / \partial \bar{u}_{-1}$, применяя его к динамическим переменным, и найдем, что $a_{i}=D X D^{-1} u_{i}=0$ для любого целого $i$. Более того,

$$
p=D X D^{-1} \bar{u}_{-1}=D X f^{-1,-1}\left(u, u_{-1}, \bar{u}_{-1}\right)=D\left(\frac{\partial f^{-1,-1}\left(u, u_{-1}, \bar{u}_{-1}\right)}{\partial \bar{u}_{-1}}\right) .
$$


Аналогично можно доказать вторую формулу. В самом деле, применим оператор $D Y D^{-1}=\sum c_{i} \partial / \partial u_{i}+d \partial / \partial \bar{u}_{-1}$ к $u_{i}$ и найдем $c_{j}=D\left(Y u_{j-1}\right)=Y u_{j}$. Затем вычислим

$$
d=D Y D^{-1} \bar{u}_{-1}=f_{u}^{-1,-1}+\frac{1}{x_{-1}} f_{u_{-1}}^{-1,-1} .
$$

Поскольку $u_{-1,-1}=f^{-1,-1}\left(u, u_{-1}, \bar{u}_{-1}\right)$, получаем $u=f^{-1,-1}\left(f\left(u, u_{1}, \bar{u}_{1}\right), u_{1}, \bar{u}_{1}\right)$. Продифференцируем последнее уравнение по $\bar{u}_{1}$ и найдем, что

$$
D \bar{D}\left(\frac{\partial f^{-1,-1}}{\partial u}\right) \frac{\partial f}{\partial \bar{u}_{1}}+D \bar{D}\left(\frac{\partial f^{-1,-1}}{\partial u_{-1}}\right)=0
$$

или, что то же самое,

$$
\frac{\partial f^{-1,-1}}{\partial u}+\frac{1}{D^{-1} \bar{D}^{-1}\left(\partial f / \partial \bar{u}_{1}\right)} \frac{\partial f^{-1,-1}}{\partial u_{-1}}=0 .
$$

Теперь в силу равенства $x=\bar{D}^{-1}\left(\partial f / \partial \bar{u}_{1}\right)$, получаем, что $d=0$.

Лемма 1. Предположим, что $Z=\sum_{-\infty}^{\infty} b_{j} \partial / \partial u_{j} \in T$ удовлетворяет следующим двум условиям: $D Z D^{-1}=c Z$ для некоторой функиии с и $b_{j_{0}} \equiv 0$ для некоторого фиксированного значения $j=j_{0}$. Тогда $T=0$.

ДоказАтельство. Имеем

$$
D Z D^{-1}=\sum_{-\infty}^{\infty} D\left(b_{j-1}\right) \frac{\partial}{\partial u_{j}}=\sum_{-\infty}^{\infty} c b_{j} \frac{\partial}{\partial u_{j}} .
$$

Если $c=0$, то $D\left(b_{j-1}\right)=0$ для любого $j$, и лемма доказана. Если $c \neq 0$, то положим $b_{j_{0}-k}=c D^{-k} b_{j_{0}}$ и $b_{j_{0}+k}=D^{k} b_{j_{0}} / c$ при $k>0$, что завершает доказательство.

\section{3. ДИСКРЕТНОЕ ПОТЕНЦИИРОВАННОЕ УРАВНЕНИЕ КДФ}

В данном разделе дается полное описание пробного кольца Ли $T$ для дискретного потенциированного уравнения КдФ [18]

$$
u_{1,1}=u+\frac{1}{u_{1}-\bar{u}_{1}}
$$

которое представляет собой очень хорошо известный пример интегрируемой модели вида (1). В силу инвариантности уравнения относительно замены $m \leftrightarrow n$ ко́льца $T$ и $\bar{T}$ должны быть изоморфны. Для этого уравнения множители $x$ и $p$ в формуле (5) одинаковые: $p=x=\left(u_{1}-\bar{u}_{-1}\right)^{2}$. Определим последовательность векторных полей

$$
\begin{aligned}
& R_{1}=[X, Y], \quad P_{1}=\left[X, R_{1}\right], \quad Q_{1}=\left[Y, R_{1}\right], \\
& R_{k+1}=\left[X, Q_{k}\right], \quad P_{k}=\left[X, R_{k}\right], \quad Q_{k}=\left[Y, R_{k}\right], \quad k \geqslant 1 \text {. }
\end{aligned}
$$

Теорема 1. Последовательность $X, Y, R_{1}, P_{1}, Q_{1}, R_{2}, P_{2}, Q_{2}, \ldots$ образует базис характеристического кольща $T$ уравнения (6). 
ДокАЗАтЕЛЬСтво. Нетрудно проверить, что $X x=-2 \sqrt{x}, Y x=2 x \sqrt{x}, X y=$ $-2 y \sqrt{y}, Y y=2 \sqrt{y}$, где $y:=D^{-1} x=x_{-1}$. Используя соотношения $D X D^{-1}=x X$ и $D(y Y) D^{-1}=Y$, можно вывести уравнения

$$
\begin{aligned}
D\left(R_{1}-2 \sqrt{y} Y\right) D^{-1} & =R_{1}-2 \sqrt{x} X, \\
D\left(P_{1}-2 \sqrt{y} R_{1}+2 y Y\right) D^{-1} & =x\left(P_{1}+2 X\right), \\
D\left(y\left(Q_{1}-2 Y\right)\right) D^{-1} & =Q_{1}+2 \sqrt{x} R_{1}-2 x X .
\end{aligned}
$$

Аналогичные формулы для $R_{2}, P_{2}$ и $Q_{2}$ имеют вид

$$
\begin{aligned}
D\left(R_{2}-2 \sqrt{y} Q_{1}\right) D^{-1} & =R_{2}+2 \sqrt{x} P_{1}, \\
D\left(P_{2}+2 \sqrt{y} R_{2}-2 y Q_{1}\right) D^{-1} & =x\left(P_{2}-2 P_{1}\right), \\
D\left(y\left(Q_{2}-2 Q_{1}\right)\right) D^{-1} & =Q_{2}+2 \sqrt{x} R_{2}+2 x P_{1} .
\end{aligned}
$$

Можно доказать по индукции, что при любом $j>1$ выполнены соотношения

$$
\begin{aligned}
D\left(R_{j}-2 \sqrt{y} Q_{j-1}\right) D^{-1} & =R_{j}+2 \sqrt{x} P_{j-1}, \\
D\left(P_{j}+2(-1)^{j} \sqrt{y} R_{j}+2(-1)^{j-1} y Q_{j-1}\right) D^{-1} & =x\left(P_{j}-2 P_{j-1}\right), \\
D\left(y\left(Q_{j}-2 Q_{j-1}\right)\right) D^{-1} & =Q_{j}+2 \sqrt{x} R_{j}-2 x P_{j-1} X .
\end{aligned}
$$

Тогда $\left[X, P_{j}\right]=0,\left[Y, Q_{j}\right]=0,\left[Y, P_{j}\right]=\left[X, Q_{j}\right],\left[R_{j}, P_{k}\right]=P_{k+j},\left[R_{j}, Q_{k}\right]=-Q_{k+j}$, $\left[R_{j}, R_{k}\right]=0,\left[P_{j}, Q_{k}\right]=-R_{k+j+1},\left[P_{j}, P_{k}\right]=0,\left[Q_{j}, Q_{k}\right]=0$.

Зададим матричное представление алгебры Ли, порожденной теми же операторами $X, Y$. Пусть $X \rightarrow \lambda \sigma_{+}, Y \rightarrow \lambda \sigma_{-}, R_{1} \rightarrow \lambda^{2} \sigma_{3}$, тогда

$$
P_{k} \rightarrow-2^{k} \lambda^{2 k+1} \sigma_{+}, \quad Q_{k} \rightarrow 2^{k} \lambda^{2 k+1} \sigma_{-}, \quad R_{k} \rightarrow 2^{k-1} \lambda^{2 k} \sigma_{3} .
$$

Здесь

$$
\sigma_{+}=\left(\begin{array}{cc}
0 & 1 \\
0 & 0
\end{array}\right), \quad \sigma_{-}=\left(\begin{array}{cc}
0 & 0 \\
1 & 0
\end{array}\right), \quad \sigma_{3}=\left(\begin{array}{cc}
1 & 0 \\
0 & -1
\end{array}\right) .
$$

Нетрудно проверить, что для уравнения (6) функция $\Delta=\Delta(k)$ является периодической с периодом $2: \Delta(2 k)=1, \Delta(2 k+1)=2$.

\section{4. УРАВНЕНИЯ ВИДА $u_{1,1}=u+g\left(u_{1}-\bar{u}_{1}\right)$}

Применим сформулированную выше гипотезу (алгебраический тест) к следующему частному классу дискретных моделей (1):

$$
u_{1,1}=u+g\left(u_{1}-\bar{u}_{1}\right)
$$

Здесь $g$ - функция, подлежащая определению.

Схема классификации. Рассмотрим следующие четыре различных случая уравнения (8) по отдельности:

$$
\begin{gathered}
\Delta(0)<\Delta_{\max }(0)=1 ; \\
\Delta(0)=\Delta_{\max }(0), \quad \Delta(1)<\Delta_{\max }(1)=2 ; \\
\Delta(0)=\Delta_{\max }(0), \quad \Delta(1)=\Delta_{\max }(1), \quad \Delta(2)<\Delta_{\max }(2)=3 ;
\end{gathered}
$$




$$
\begin{gathered}
\Delta(0)=\Delta_{\max }(0), \quad \Delta(1)=\Delta_{\max }(1), \quad \Delta(2)=\Delta_{\max }(2), \\
\Delta(k) \leqslant 1 \quad \text { при некотором } \quad k>2 .
\end{gathered}
$$

Здесь $\Delta_{\max }(k)$ обозначает наибольшее значение функции $\Delta(k)$ для уравнения $(1)$, когда $f\left(u, u_{1}, \bar{u}_{1}\right)$ пробегает класс произвольных функций.

Удивительно, что для уравнения (8) исследование первых трех частных случаев (9а)-(9в) позволяет выделить весьма короткий список уравнений, для которых можно ожидать, что они будут интегрируемыми. Этот список исчерпывающий, поскольку случай $(9 г)$ никогда не реализуется (см. ниже следствия 1 и 2 из теорем 2 и 3 соответственно).

Введем векторные поля $R_{1}, P_{1}, Q_{1}$ и $R_{2}$ по формулам (7), а также поля $W=$ $\left[Y, Q_{1}\right], Z=\left[X, P_{1}\right]$. Используя их, можно в дополнение к $V_{0}$ и $V_{1}$ ввести еще два линейных пространства

$$
V_{2}=V_{1}+\left\{P_{1}, Q_{1}\right\}, \quad V_{3}=V_{2}+\left\{W, Z, R_{2}\right\}
$$

Чтобы вычислить $\Delta(k)$, будем использовать автоморфизм (4). Определим множители $x$ и $p$ в формуле (5) в случае модели (8): $x=p=-g^{\prime}\left(g^{-1}\left(u_{1}-\bar{u}_{-1}\right)\right)$, где функция $\beta=g^{-1}(\alpha)$ является обратной к функции $\alpha=g(\beta)$. Наоборот, зная $x=x\left(u_{1}-\bar{u}_{-1}\right)$, можно воспроизвести $g(\beta)$, используя уравнение

$$
\beta=g^{-1}(\alpha)=\int\left(g^{-1}(\alpha)\right)^{\prime} d \alpha=\int \frac{d \alpha}{g^{\prime}\left(g^{-1}(\alpha)\right)}=-\int \frac{d \alpha}{x(\alpha)} .
$$

Определим действие операторов $X$ и $Y$ на переменную $x$. Очевидно, $X x=-x^{\prime}$, $Y x=x x^{\prime}$. Прямое вычисление дает

$$
\begin{aligned}
D R_{1} D^{-1} & =R_{1}+\frac{x^{\prime}}{x} Y-x^{\prime} X \\
D P_{1} D^{-1} & =x P_{1}+x^{\prime} R_{1}-r Y+x r X, \quad r=x^{\prime \prime}-\frac{\left(x^{\prime}\right)^{2}}{x}, \\
D Q_{1} D^{-1} & =\frac{1}{x} Q_{1}+\frac{x^{\prime}}{x} R_{1}+\frac{x^{\prime \prime}}{x} Y-x^{\prime \prime} X, \\
D W D^{-1} & =\frac{1}{x^{2}} W+\left(\frac{2 x^{\prime \prime}}{x}-\frac{\left(x^{\prime}\right)^{2}}{x^{2}}\right) R_{1}+\frac{x^{\prime \prime \prime}}{x} Y-x^{\prime \prime \prime} X, \\
D Z D^{-1} & =x^{2} Z+\left(\left(x^{\prime}\right)^{2}-2 x x^{\prime \prime}\right) R_{1}+q Y-x q X, \quad q=x x^{\prime \prime \prime}-2 x^{\prime} x^{\prime \prime}+\frac{\left(x^{\prime}\right)^{3}}{x}, \\
D R_{2} D^{-1} & =R_{2}+\frac{x^{\prime}}{x} Q_{1}+x^{\prime} P_{1}+\frac{\left(x^{\prime}\right)^{2}}{x} R_{1}-s Y+x s X, \quad s=x^{\prime \prime \prime}-\frac{x^{\prime} x^{\prime \prime}}{x} .
\end{aligned}
$$

Исследуем множество $G$ всех кратных коммутаторов $X$ и $Y$.

ЛЕмма 2. Коэффициенты любого оператора из множества $G$ являются функииями от конечного числа переменных $x, x_{ \pm 1}, x_{ \pm 2}, \ldots$.

ДокАЗАТЕЛьство. Поскольку $x=x\left(u_{1}-\bar{u}_{-1}\right)$, можно записать $x^{\prime}=\phi(x)$ для некоторой функции $\phi$. Тогда $X(x)=-\phi(x)$ и $Y(x)=x \phi(x)=: \psi(x)$. Используя соотношения сопряжения $D X D^{-1}=x X$ и $D Y D^{-1}=(1 / x) Y$, получаем равенства $X\left(x_{j}\right)=\phi^{j}\left(x, x_{1}, \ldots, x_{j}\right)$ и $Y\left(x_{j}\right)=\psi^{j}\left(x, x_{1}, \ldots, x_{j}\right)$. Аналогично, имеем равенства 
$X\left(x_{-j}\right)=\phi^{-j}\left(x, x_{-1}, \ldots, x_{-j}\right)$ и $Y\left(x_{-j}\right)=\psi^{-j}\left(x, x_{-1}, \ldots, x_{-j}\right)$. Теперь очевидно, что функция

$$
R_{1}=X(x) \frac{\partial}{\partial u_{1}}+X\left(\frac{1}{x_{-1}}\right) \frac{\partial}{\partial u_{-1}}+X\left(x x_{1}\right) \frac{\partial}{\partial u_{2}}+\cdots
$$

удовлетворяет утверждению леммы. Из формул $R_{1}(x)=X(x) x^{\prime}$ и $D R_{1} D^{-1}=$ $R_{1}+\left(x^{\prime} / x\right) Y-x^{\prime} X$ получаем $R_{1}\left(x_{j}\right)=\rho^{j}\left(x, x_{1}, \ldots, x_{j}\right)$. Ясно, что дальнейшее доказательство леммы можно провести по индукции.

Теорема 2. Если цепочка вида (8) удовлетворяет одному из условий (9а)-(9в) в схеме классификации, то функиия $x=x(\alpha)$ есть решение обыкновенного дифференииального уравнения

$$
\left(x^{\prime}\right)^{2}=\left(x^{2}+1\right) \gamma+x \nu, \quad \gamma, \nu=\text { const } .
$$

ДокАЗАТЕЛЬСтво. Начнем со случая (9а) и предположим, что $\Delta(0)=0$; тогда имеем $R_{1}=\lambda X+\mu Y$. Очевидно, что $R_{1}=X(x) \partial / \partial u_{1}+\cdots$, поэтому $\lambda=\mu=0$, откуда следует, что $R_{1}=0$. Применяя указанный выше автоморфизм к обеим частям уравнения (12), получаем $\left(x^{\prime} / x\right) Y-x^{\prime} X=0$. Поскольку $X$ и $Y$ линейно независимы, отсюда имеем уравнение $x^{\prime}=0$, которое представляет собой частный случай уравнения (12). Очевидно, его решением является $x=c$, и с учетом (10) мы получаем $\beta=g^{-1}(\alpha)=-(1 / c) \alpha+c_{1}$. Таким образом, наше уравнение $\alpha=g(\beta)$ (см. формулу (8)) является линейным, $u_{1,1}-u=-c\left(u_{1}-\bar{u}_{1}+c_{1}\right)$. При этом $\operatorname{dim} T=2$, так что $\Delta(k)=0$ при $k \geqslant 0$.

Аналогично проверяем, что условие (9б) приводит к уравнению (12). Действительно, предположим, что $\Delta(0)=1$ и $\Delta(1)<2$. Тогда имеем

$$
P_{1}=\nu Q_{1}+\epsilon R_{1}
$$

Здесь в силу леммы 2 функции $\nu$ и $\epsilon$ могут зависеть только от $x, x_{ \pm 1}, x_{ \pm 2}, \ldots$ Применим автоморфизм (4) к обеим частям уравнения (13) и затем упростим с учетом уравнений (11):

$$
\begin{aligned}
& x\left(\nu Q_{1}+\epsilon R_{1}\right)+x^{\prime} R_{1}-r Y+x r X= \\
& \quad=D(\nu)\left(\frac{1}{x} Q_{1}+\frac{x^{\prime}}{x} R_{1}+\frac{x^{\prime \prime}}{x} Y-x^{\prime \prime} X\right)+D(\epsilon)\left(R_{1}+\frac{x^{\prime}}{x} Y-x^{\prime} X\right) .
\end{aligned}
$$

Из сравнения коэффициентов перед линейно независимыми операторами получаем условия

$$
\begin{aligned}
Q_{1}: & x \nu=\frac{1}{x} D(\nu), \\
R_{1}: & x^{\prime}+x \epsilon=\frac{x^{\prime}}{x} D(\nu)+D(\epsilon), \\
Y: & r=\frac{x^{\prime}}{x} D(\epsilon)+\frac{x^{\prime \prime}}{x} D(\nu), \\
X: & x r=-x^{\prime} D(\epsilon)-x^{\prime \prime} D(\nu) .
\end{aligned}
$$


Анализируя эти равенства, приходим к выводу, что уравнение (13) выполнено, если и только если справедливы следующие три условия: $\nu=0, \epsilon=$ const, $x^{\prime}=$ $\epsilon(1-x)$. Действительно, при этих условиях два последних уравнения выполняются автоматически. Аналогично можно проверить, что $Q_{1}=\nu P_{1}-\epsilon R_{1}$ эквивалентно тем же трем условиям. Таким образом, если $\Delta(1)<2$, то $\Delta(1)=0$, и поэтому $\Delta(k)=0$ для любого натурального $k \geqslant 1$. В этом случае $\operatorname{dim} T=3$.

Теперь предположим, что $\Delta(0)=1, \Delta(1)=2$ и $\Delta(2) \leqslant 2$, что соответствует случаю (9в). Пусть $Z$ линейно выражается через другие векторные поля из $V_{3}$ :

$$
Z=\alpha X+\beta Y+\gamma R_{1}+\delta P_{1}+\epsilon Q_{1}+\phi R_{2}+\psi W .
$$

Очевидно, $\alpha=\beta=0$, поскольку $X=\partial / \partial \bar{u}_{-1}$ и $Y=\partial / \partial u+x \partial / \partial u_{1}+\cdots$, а $Z$ не содержит членов $\partial / \partial \bar{u}_{-1}$ и $\partial / \partial u$.

Применяя автоморфизм к обеим частям равенства (14) и сравнивая коэффициенты перед линейно независимыми операторами, находим

$$
\begin{array}{ll}
W: & x^{2} \psi=D(\psi) \frac{1}{x^{2}}, \\
R_{2}: & x^{2} \phi=D(\phi), \\
Q_{1}: & x^{2} \epsilon=D(\epsilon) \frac{1}{x}+\frac{x^{\prime}}{x} \phi, \\
P_{1}: & x^{2} \delta=D(\delta) x+x^{\prime} \phi, \\
R_{1}: & x^{2} \gamma+\left(x^{\prime}\right)^{2}-2 x x^{\prime \prime}=D(\delta) x^{\prime}+D(\gamma) .
\end{array}
$$

Поскольку $x=x\left(u_{1}-\bar{u}_{-1}\right)$, имеем $\psi=0, \phi=0, \epsilon=0, \delta=0, \gamma=$ const. Cравнение коэффициентов при $X$ и $Y$ дает еще одно уравнение $x q=\gamma x^{\prime}$. Наконец, получаем два обыкновенных дифференциальных уравнения для $x$ :

$$
x^{2} x^{\prime \prime \prime}-2 x x^{\prime} x^{\prime \prime}+\left(x^{\prime}\right)^{3}=\gamma x^{\prime}, \quad\left(x^{2}-1\right) \gamma+\left(x^{\prime}\right)^{2}-2 x x^{\prime \prime}=0 .
$$

Условие совместности этих уравнений эквивалентно уравнению (12). В этом случае $Z=\gamma R_{1}$. Замечательно, что $x$ есть решение уравнения (12), если и только если $W$ линейно выражается через другие элементы из $V_{3}$, и тогда $W=\gamma R_{1}$. И последняя возможность - поле $R_{2}$ линейно выражается через $X, Y, R_{1}, P_{1}, Q_{1}, W$ и $Z$. В этом случае $x$ является решением уравнения $x^{\prime}=0$. Доказательство теоремы завершено.

Чтобы найти $x=x(\alpha)$, вычислим интеграл

$$
H(x):=\int \frac{d x}{\sqrt{\left(x^{2}+1\right) \gamma+x \nu}}=\frac{1}{\sqrt{\gamma}} \ln \left(2 \sqrt{\left(x^{2}+1\right) \gamma+x \nu}+2 x+b\right) .
$$

Теперь найдем $x$, решая уравнение $H(x)=\alpha-\alpha_{0}$ :

$$
x(\alpha)=\frac{1}{4} e^{\sqrt{\gamma}\left(\alpha-\alpha_{0}\right)}-\frac{\nu}{2 \gamma}-\left(1-\frac{\nu^{2}}{4 \gamma^{2}}\right) e^{-\sqrt{\gamma}\left(\alpha-\alpha_{0}\right)} .
$$

Чтобы получить соответствующее уравнение (8) на квад-графе, проинтегрируем еще раз:

$$
\beta=g^{-1}(\alpha)=-\int \frac{d \alpha}{x(\alpha)}
$$


Тогда искомое уравнение будет иметь вид (8). Наша гипотеза состоит в том, что если уравнение (8) является $S$-интегрируемым, то $g$ получается из соотношений $(15),(16)$. Вычислив интеграл (16), находим список требуемых уравнений.

Из теоремы 2 вытекает следующее утверждение.

СлЕДСТвИЕ 1. Если для иепочки (8) выполнено одно из условий (9а)-(9в), то цепочка имеет один из следующих видов:

$$
\begin{array}{lll}
u_{1,1}-u=c\left(u_{1}-\bar{u}_{1}-\beta_{0}\right) & n p u & \gamma=\nu=0 ; \\
a e^{\sqrt{\gamma}\left(u_{1,1}-u\right)}=b+c e^{-\sqrt{\gamma}\left(u_{1}-\bar{u}_{1}\right)} & n p u & \nu=-2 \gamma ; \\
\left(u_{1,1}-u-\alpha_{0}\right)\left(u_{1}-\bar{u}_{1}-\beta_{0}\right)=\frac{1}{4 \nu} & n p u & \gamma=0, \quad \nu \neq 0 ; \\
a e^{\sqrt{\gamma}\left(u_{1,1}-u\right)}=b+c e^{\sqrt{\gamma}\left(u_{1}-\bar{u}_{1}\right)} & n p u & \nu=2 \gamma ; \\
\left(4-\frac{\nu^{2}}{\gamma^{2}}\right) e^{\sqrt{\gamma}\left(u_{1,1}-u-\alpha_{0}\right)}+\frac{\nu}{\gamma}=2 \operatorname{th} \frac{\sqrt{\gamma}\left(u_{1}-\bar{u}_{1}\right)-\beta_{0}}{2} & n p u \quad \gamma \neq 0, \quad \nu \neq \pm 2 \gamma
\end{array}
$$

где $a, b, c, \alpha_{0}, \beta_{0}-$ константы.

Уравнение (19а) есть не что иное, как хорошо известное дискретное потенциированное уравнение КдФ (6). Уравнения (18) и (19б) сводятся одно к другому простой заменой независимых переменных $m \leftrightarrow n$. Сделав замену переменных $u=(1 / \sqrt{\gamma}) \ln v$, мы приводим два последних уравнения (19б) и (19в) к билинейному виду

$$
\begin{gathered}
a \bar{v}_{1} v_{1,1}=b v \bar{v}_{1}+c v v_{1}, \\
\left(\alpha_{1} v_{1}+\alpha_{2} \bar{v}_{1}\right) v_{1,1}+v\left(\alpha_{3} v_{1}+\alpha_{4} \bar{v}_{1}\right)=0
\end{gathered}
$$

где

$$
\begin{aligned}
\alpha_{1} & =\left(2-\frac{\nu^{2}}{2 \gamma^{2}}\right) e^{-\sqrt{\gamma} \alpha_{0}-\beta_{0}} \neq 0, & \alpha_{2} & =\left(2-\frac{\nu^{2}}{2 \gamma^{2}}\right) e^{-\sqrt{\gamma} \alpha_{0}} \neq 0, \\
\alpha_{3} & =\left(\frac{\nu}{2 \gamma}-1\right) e^{-\beta_{0}} \neq 0, & \alpha_{4} & =\left(\frac{\nu}{2 \gamma}+1\right) \neq 0 .
\end{aligned}
$$

Уравнение (20а) было введено в работе [11], а недавно было доказано, что оно допускает бесконечный ряд высших симметрий [19]. Уравнение (20б) представляет собой частный случай уравнения Хиетаринта-Виалле [20]

$$
c_{1} u \bar{u}_{1}+c_{2} u_{1} u_{1,1}+c_{3} u u_{1}+c_{4} \bar{u}_{1} u_{1,1}+c_{5}\left(u u_{1,1}+u_{1} \bar{u}_{1}\right)=0
$$

при $c_{5}=0$. Недавно было доказано, что это уравнение проходит симметрийный тест [21].

Исследуем множество $G$, состоящее из полей $X, Y$ и всех их кратных коммутаторов. Определим порядок ord $Z$ элемента $Z \in G$ как число входящих в него $X$ и $Y$ минус единица. Например, $\operatorname{ord}[X, Y]=1, \operatorname{ord}[X,[X, Y]]=2$ и т. д. Определим степень $\operatorname{deg} Z$ элемента $Z$ как показатель $k$ в соотношении сопряжения $D Z D^{-1}=$ 
$x^{k} Z+\cdots$, где многоточием обозначена линейная комбинация элементов с порядком, меньшим чем ord $Z$. Обозначим через $G_{i, j}$ подмножество в $G$, содержащее элементы с порядком $i$ и степенью $j$. Рассмотрим объединение $G_{i}=\bigcup_{j} G_{i, j}$. Очевидно, что множество $G_{i, i-1}$ (множество $G_{i,-i+1}$ ) содержит единственный элемент $Z_{i, i-1}=\operatorname{ad}_{X}^{i}(Y)$ с точностью до множителя -1 (соответственно единственный элемент $Z_{i,-i+1}=\operatorname{ad}_{Y}^{i}(X)$ с точностью до множителя -1$)$. Здесь оператор ad определяется как $\operatorname{ad}_{X}(Y)=[X, Y]$.

ТЕОрема 3. Пусть операторы $Z_{k, k-1}$ (операторы $Z_{k,-k+1}$ ) лежат в базисе линейного пространства $V_{k} \supset G_{k}$ при всех $k, 3 \leqslant k<N$, а оператор $Z_{N, N-1}$ (coответственно $Z_{N,-N+1}$ ) линейно выражается через другие операторы в $V_{N}$. Тогда бункция $x=x\left(u_{1}-\bar{u}_{-1}\right)$ является решением уравнения вида $x^{\prime}=\epsilon(x-1)$ с постоянным коэффициентом $\epsilon$.

Доказательству теоремы предпошлем следующую лемму.

ЛЕмма 3. При любом $k \geqslant 3$ справедливо равенство

$$
D Z_{k+1, k} D^{-1}=x^{k} Z_{k+1, k}-c_{k} x^{\prime} x^{k-1} Z_{k, k-1}+\cdots,
$$

где $c_{k} \geqslant 0$ (причем $c_{k}>0$ при $\left.k>3\right)$, а многоточием обозначена линейная комбиначия операторов порядка менъше $k$.

ДокАЗАТЕЛЬство. Докажем лемму по индукции. Из уравнений (11) получаем для $Z=Z_{3,2}$ и $R_{1}=Z_{1,0}$ представление

$$
D Z_{3,2} D^{-1}=x^{2} Z_{3,2}+\left(\left(x^{\prime}\right)^{2}-2 x x^{\prime \prime}\right) Z_{1,0}+q Y-x q X, \quad q=x x^{\prime \prime \prime}-2 x^{\prime} x^{\prime \prime}+\frac{\left(x^{\prime}\right)^{3}}{x},
$$

показывающее, что утверждение верно при $k=3$. Теперь предположим, что

$$
D Z_{k, k-1} D^{-1}=x^{k-1} Z_{k, k-1}-c_{k-1} x^{\prime} x^{k-2} Z_{k-1, k-2}+\cdots,
$$

и вычислим $D Z_{k+1, k} D^{-1}$ :

$$
\begin{aligned}
D Z_{k+1, k} D^{-1} & =\left[x X, x^{k-1} Z_{k, k-1}-c_{k-1} x^{\prime} x^{k-2} Z_{k-1, k-2}+\cdots\right]= \\
& =x^{k} Z_{k+1, k}-c_{k} x^{\prime} x^{k-1} Z_{k, k-1}+\cdots,
\end{aligned}
$$

где $c_{k}=c_{k-1}+k-1>c_{k-1}>0$. Доказательство леммы завершено.

ДоКАЗАТЕЛЬСТВо тЕОРЕМЫ 3. Предположим, что

$$
Z_{N, N-1}=\sum_{\text {ord } Z_{\nu}=N} a_{\nu} Z_{\nu}+\sum_{\text {ord } Z_{\mu}=N-1} b_{\mu} Z_{\mu}+\cdots,
$$

где $Z_{\nu}$ и $Z_{\mu}$ - базис в $V_{N}$, а многоточием обозначена линейная комбинация операторов меньшего порядка. Применим автоморфизм $D(\cdot) D^{-1}($ см. формулу (4)) к обеим частям равенства (21):

$$
\begin{aligned}
x^{N-1} & \left(\sum_{\operatorname{ord} Z_{\nu}=N} a_{\nu} Z_{\nu}+\sum_{\operatorname{ord} Z_{\mu}=N-1} b_{\mu} Z_{\mu}+\cdots\right)-x^{N-2} x^{\prime} c_{N-1} Z_{N-1, N-2}+\cdots= \\
& =\sum_{\operatorname{ord} Z_{\nu}=N} D\left(a_{\nu}\right)\left(x^{k_{\nu}} Z_{\nu}+\cdots\right)+\sum_{\text {ord } Z_{\mu}=N-1} D\left(b_{\mu}\right)\left(x^{k_{\mu}} Z_{\mu}+\cdots\right) .
\end{aligned}
$$


Сравнивая коэффициенты перед $Z_{\nu}$, получаем

$$
x^{N-1} a_{\nu}=x^{k_{\nu}} D\left(a_{\nu}\right), \quad k_{\nu} \neq N-1 .
$$

В силу леммы 2 функции $a_{\nu}$ и $b_{\mu}$ зависят от переменной $x$ и ее сдвигов. С другой стороны, из формулы (22) следует, что функции $a_{\nu}$ не могут зависеть от $x, x_{ \pm 1}, x_{ \pm 2}, \ldots$. Поэтому единственной возможностью является $a_{\nu}=0$. Теперь, сравнивая коэффициенты перед $Z_{N-1, N-2}$, находим

$$
x^{N-1} b-c_{N-1} x^{N-2} x^{\prime}=x^{N-2} D(b),
$$

где $b$ - коэффициент при $Z_{N-1, N-2}$ в разложении (21). Простой анализ уравнения (23) показывает, что $b$ - константа. Таким образом, уравнение (23) эквивалентно уравнению $x^{\prime}=\epsilon(x-1)$ при $\epsilon=b / c_{N-1}$. Теорема доказана.

СЛЕДСтвиЕ 2. Случай (9г) схемы классификации никогда не реализуется.

ДокАЗАТЕЛЬство. Предположим противное: пусть данный случай реализуется. Тогда по крайней мере одно из векторных полей $Z_{k, k-1}$ или $Z_{k,-k+1}$ должно линейно выражаться через другие элементы из $V_{k}$, иначе $\Delta(k) \geqslant 2$. Поэтому в силу теоремы 3 мы имеем $x^{\prime}=\epsilon(x-1)$, что соответствует случаю (9б) $\Delta(0)=1, \Delta(1)<2$. Полученное противоречие показывает, что наше допущение неверно. Доказательство завершено.

Рассмотрим подробно пробные кольца $T$ и $\bar{T}$ для уравнения (19a) из списка в следствии 1. Ранее было отмечено, что это уравнение редуцируется к уравнению (18) простой заменой независимых переменных $m \leftrightarrow n$. Как было показано выше в случае уравнения (18), размерность кольца $T$ равна 3 . Отсюда немедленно следует, что для уравнения (19б) мы имеем $\operatorname{dim} \bar{T}=3$. Теперь сосредоточимся на кольце $T$ для этого уравнения. Без потери общности положим $\gamma=1$ и получим

$$
a e^{u_{1,1}-u}=b+c e^{u_{1}-\bar{u}_{1}} .
$$

Мы уже доказали, что для уравнения (24) функция $x=x(\alpha)$ является решением уравнения $x^{\prime}=x+1$. Характеристические векторные поля $X$ и $Y$ действуют на переменные $x, y=D^{-1} x$ следующим образом:

$X x=-x^{\prime}=-x-1, \quad X y=-y y^{\prime}=-y^{2}-y, \quad Y x=x x^{\prime}=x^{2}+x, \quad Y y=y+1$.

Теорема 4. Последовательность $X, Y, R_{1}, P_{1}, Q_{1}, R_{2}, P_{2}, Q_{2}, \ldots$ определена как $R_{1}=[X, Y], P_{1}=\left[X, R_{1}\right], Q_{1}=\left[Y, R_{1}\right], \ldots, R_{k+1}=\left[X, Q_{k}\right], P_{k}=\left[X, R_{k}\right], Q_{k}=\left[Y, R_{k}\right]$, $k \geqslant 1$, и образует базис пробного кольца $T$ уравнения (24).

СХЕмА ДОКАЗАТЕЛЬСТВА. Начнем с соотношений сопряжения для кратных коммутаторов. Например, $D\left(R_{1}-Y+X\right) D^{-1}=R_{1}+Y-X$. Нетрудно доказать по индукции, что при $k \geqslant 1$

$$
\begin{aligned}
D\left(P_{k}-R_{k}\right) D^{-1} & =x\left(P_{k}+R_{k}\right), \\
D\left(Q_{k}-R_{k}\right) D^{-1} & =\frac{1}{x}\left(Q_{k}+R_{k}\right), \\
D\left(R_{k+1}-Q_{k}-P_{k}+R_{k}\right) D^{-1} & =R_{k+1}+Q_{k}+P_{k}+R_{k} .
\end{aligned}
$$


Из этих соотношений следует, что $\left[Y, P_{k}\right]=\left[X, Q_{k}\right]$ и $\left[X, P_{1}\right]=\left[Y, Q_{1}\right]=R_{1}$. Тогда получаем

$$
\begin{aligned}
& {\left[R_{k}, R_{j}\right]=\left[Q_{k}, Q_{j}\right]=\left[P_{k}, P_{j}\right]=0,} \\
& {\left[R_{k}, P_{j}\right]=P_{k+j}-Q_{k+j-1}, \quad\left[R_{k}, Q_{j}\right]=-Q_{k+j}+P_{k+j-1},} \\
& {\left[P_{k}, Q_{j}\right]=-R_{k+j+1}+R_{k+j-1} .}
\end{aligned}
$$

Теперь можно заключить, что для уравнения (24) пробное кольцо $T$ удовлетворяет условиям $\Delta(2 k)=1$ и $\Delta(2 k+1)=2$, равно как и для дискретного потенциированного уравнения КдФ (19a). Таким образом, это уравнение полностью проходит наш алгебраический тест. Для случая (19в) в списке из следствия 1 пробные кольца пока подробно не исследовались.

Суммируем полученные выше результаты в следующей теореме.

Теорема 5. Предположим, что уравнение вида (8) проходит алгебраический тест (удовлетворяет гипотезе из раздела 2). Тогда оно имеет вид, заданный в следствии 1 из теоремь 2.

\section{5. ЗАКЛЮЧЕНИЕ}

Мы предложили новую схему классификации уравнений на квад-графах, основанную на распределениях характеристических векторных полей (см., например, [22]). Получен исчерпывающий список уравнений вида $u_{1,1}-u=g\left(u_{1}-\bar{u}_{1}\right)$, проходящих предложенный тест на интегрируемость. Важно, что обо всех найденных уравнениях уже известно, что они интегрируемы. Показано, что выдвинутая гипотеза является эффективным средством классификации интегрируемых уравнений на квад-графах.

Благодарности. Авторы выражают благодарность А. В. Жиберу и Р. И. Ямилову за полезные обсуждения. Настоящая работа выполнена при частичной финансовой поддержке РФФИ (гранты № 10-01-91222-CT_a, 11-01-97005-r_povoljie_a, 10-01-00088_a).

\section{Список литературы}

[1] A. I. Bobenko, Yu. B. Suris, Int. Math. Res. Not., 2002:11 (2002), 573-611, arXiv: nlin/0110004.

[2] F. W. Nijhoff, Phys. Lett. A, 297:1-2 (2002), 49-58, arXiv: nlin/0110027.

[3] V. E. Adler, A. I. Bobenko, Yu. B. Suris, Comm. Math. Phys., 233:3 (2003), 513-543, arXiv: nlin/0202024.

[4] F. W. Nijhoff, A. J. Walker, Glasgow Math. J., 43:A (2001), 109-123.

[5] D. Levi, R. I. Yamilov, On a nonlinear integrable difference equation on the square 3D-inconsistent, arXiv: 0902.2126; J. Nonlinear Math. Phys., 11:1 (2004), 75-101.

[6] P. Xenitidis, Integrability and symmetries of difference equations: the Adler-Bobenko-Suris case, arXiv: 0902.3954.

[7] O. G. Rasin, P. E. Hydon, J. Phys. A, 40:42 (2007), 12763-12773.

[8] A. V. Mikhailov, J.P. Wang, P. Xenitidis, Recursion operators, conservation laws and integrability conditions for difference equations, arXiv: 1004.5346.

[9] A. Tongas, D. Tsoubelis, P. Xenitidis, J. Math. Phys., 42:12 (2001), 5762-5784. 
[10] M.P. Bellon, C.-M. Viallet, Comm. Math. Phys., 204:2 (1999), 425-437, arXiv: chao-dyn/9805006.

[11] F. W. Nijhoff, A. Ramani, B. Grammaticos, Y. Ohta, Stud. Appl. Math., 106:3 (2001), 261-314, arXiv: solv-int/9812011.

[12] B. Grammaticos, G. Karra, V. Papageorgiou, A. Ramani, "Integrability of discrete-time systems", Chaotic Dynamics, NATO Adv. Sci. Inst. Ser. B. Phys., 298, ed. T. Bountis, Plenum, New York, 1992, 75-90.

[13] J. Hietarinta, J. Nonlinear Math. Phys., 12, Suppl. 2 (2005), 223-230.

[14] А. Н. Лезнов, В. Г. Смирнов, А. Б. Шабат, ТМФ, 51:1 (1982), 10-21.

[15] А. В. ЖКибер, Ф. Х. Мукминов, "Квадратичные системы, симметрии, характеристические и полные алгебры", Задачи математической физики и асимптотика их решений, ред. Л. А. Калякин, БНЦ УРО АН СССР, Уфа, 1991, 14-33.

[16] А. В. Жибер, Р. Д. Муртазина, Фундамент. и прикл. матем., 12:7 (2006), 65-78.

[17] I. T. Habibullin, SIGMA, 1 (2005), 023, 9 pp., arXiv: nlin.SI/0506027.

[18] F. W. Nijhoff, H. W. Capel, Acta Appl. Math., 39:1-3 (1995), 133-158.

[19] A. G. Rasin, J. Phys. A, 43:23 (2010), 235201, 11 pp., arXiv: 1001.0724.

[20] J. Hietarinta, C. Viallet, J. Phys. A, 40:42 (2007), 12629-12643, arXiv: 0705.1903.

[21] D. Levi, R. I. Yamilov, Generalized symmetry integrability test for discrete equations on the square lattice, arXiv: 1011.0070, accepted by J. Phys. A.

[22] B. Doubrov, I. Zelenko, J. London Math. Soc., 80:3 (2009), 545-566, arXiv: math.DG/0703662. 